\title{
Developing a Nomogram to Predict the Probability of Subsequent Vascular Events at 6-Month in Chinese Patients with Minor Ischemic Stroke
}

\author{
Yuping Du' \\ Ping $\mathrm{Gu}^{2}$ \\ $\mathrm{Yu} \mathrm{Cui}{ }^{2}$ \\ Yi Wang ${ }^{2}$ \\ Juanjuan $\operatorname{Ran}^{2}$ \\ 'Department of Neurology, the 904th \\ Hospital of Joint Logistic Support Force, \\ PLA, Wuxi, 2I 4044, People's Republic of \\ China; ${ }^{2}$ Department of Neurology, Wuxi \\ No.5 People's Hospital, Wuxi, 2I4000, \\ People's Republic of China
}

Correspondence: Juanjuan Ran Department of Neurology, Wuxi No.5 People's Hospital, Wuxi, Jiangsu Province, People's Republic of China

Tel +86- | 3255109976

Fax +86-510-68585555

Email 7532164@qq.com
Purpose: To develop a nomogram to predict the risk of subsequent vascular events (SVE) at 6-month in Chinese patients with minor ischemic stroke (MIS).

Patients and Methods: We performed a retrospective analysis of 260 MIS patients, which were randomly divided into a derivation set (193 cases) and a verification set (67 cases) at a ratio of 3:1. Multi-factor logistic regression was used to construct a predictive model of SVE from the derivation set and verify it in the verification set.

Results: Finally, there were 51 cases (19.6\%) of SVE in 260 MIS cases. Age, fasting blood glucose, metabolic syndrome, number of lesions found on MRI, and the infarct size were used to construct the prediction model and nomogram. The AUC in the derivation set was 0.901 , with a sensitivity of 0.795 , a specificity of 0.877 , a positive likelihood ratio of 6.443 , and a negative likelihood ratio of 0.234 . The AUC in the verification set was 0.897 , which was not significantly different from the derivation set $(\mathrm{P}=0.937)$. The predictive model based on clinical parameters has good diagnostic efficiency and robustness.

Conclusion: The nomogram can provide personalized predictions for the 6-month SVE risk in Chinese MIS patients.

Keywords: logistic models, nomograms, brain ischemia, stroke, infarction

\section{Introduction}

There are approximately 3 million new cases of strokes in China every year, and about $30 \%$ of them are minor ischemic stroke (MIS). ${ }^{1,2}$ The lacunar stroke subtype usually indicates MIS due to small vessel disease (SVD). MIS patients had brain ischemia with a resolution of symptoms and were characterized by a National Institutes of Health Stroke Scale (NIHSS) score $\leq 3{ }^{3}$ The risk of ischemic stroke and other major vascular events shortly after a MIS is high. MIS is a serious problem that threatens human health, and it is critical to identify the patients at risk of poor outcomes after MIS in the early stage of clinical management. ${ }^{4-7}$

Many factors are reported to be closely related to subsequent vascular events (SVE) of MIS. 25(OH)D is reported to predict death and functional outcome in Chinese acute ischemic stroke patients within 90 days. ${ }^{8}$ Moreover, it has been reported that the poor outcome of MIS was related to advanced age, female, diabetes mellitus, and positive diffusion weighted imaging (DWI). ${ }^{9}$ However, a single risk factor is not accurate in predicting the incidence of SVE in MIS. Thus, multi-factor predictive models are expected to improve the predictive performance. The nomogram, one kind of multi-factor predictive model, is a pictorial 
representation of a complicated mathematical formula. It can enable users to map the subject-specific covariates to the probability of an event by graphically representing the influence of each variable on the result. ${ }^{10}$ The current prognostic prediction models of ischemic strokes (IS) mainly include the following. The NIHSS is a 15-item impairment scale that has established predictive validity for long-term stroke outcomes. ${ }^{11}$ The modified Rankin scale (mRS), a clinician-reported measure of global disability, is widely applied for evaluating recovery from stroke and as an endpoint in randomized clinical trials. ${ }^{12}$ NIHSS score on admission, Age, previous Diabetes mellitus, and crEatinine (NADE), the first nomogram developed and validated in Chinese patients with IS, can independently predict the probability of an adverse outcome in 6 months (mRS score $>2$ ). ${ }^{13}$ Creatinine, fast blood glucose, age, previous cerebral hemorrhage, previous valvular heart disease, and NHISS score (COACHS) nomogram, which can be used to predict the poor prognosis of the Chinese population at 3 months after acute ischemic stroke (mRS score $>2$ ), is also a reliable tool for effective risk stratification of acute stroke patients. ${ }^{14}$ In 2017, Gianni Turcato et $\mathrm{al}^{15}$ published a retrospective study on Italian patients with acute ischemic stroke and constructed a prediction model for the adverse outcome at a 3-month (mRS score, 3-6). NIHSS Stroke Scale score, Age, pre-stroke mRS score, onset-to-treatment Time (START) is a nomogram that predicts the poor prognosis of stroke patients who received intravenous thrombolysis, and this model has been validated in the Chinese population. ${ }^{16,17}$ In 2019, Sun et $\mathrm{al}^{18}$ conducted a retrospective study on patients with conservative large hemispheric cerebral infarction. They combined important prognostic factors and constructed a nomogram to predict each patient's risk of hospital death. However, the patient population of these studies was IS patients, which included both MIS patients and non-MIS patients, and the MIS population was not analyzed separately in these studies. ${ }^{13-17}$ Moreover, the START model was aimed to predict the poor prognosis of stroke patients who received intravenous thrombolysis, which belongs to a specific population of IS. ${ }^{16,17}$ In the study of Sun et $\mathrm{al}^{18}$ the patient population was patients with cerebral infarction in the greater hemisphere, which was different from MIS. Because MIS patients have mild symptoms that are easily ignored, the IS model may not be accurate enough to predict the prognosis of MIS patients. ${ }^{3}$ Therefore, we need a predictive model specifically for the poor prognosis of MIS patients. However, to the best of our knowledge, there are few reports about this.

This study aims to establish and verify a predictive model for the SVE risk at 6-month in Chinese MIS patients. The nomogram can help guide the risk stratification of MIS patients and treatment decisions.

\section{Patients and Methods Patients and Study Design}

In this retrospective case-control study, we initially screened 280 patients who attended the Neurology Clinic of Wuxi No.5 People's Hospital from January 2017 to December 2019. The study followed the Declaration of Helsinki and was approved by the ethics committee of the Wuxi No.5 People's Hospital (ethics number: 2020-08-001). The data were all anonymous, and thus the informed consent was not required. Moreover, the exemption for patient consent was approved by the medical officer of the Wuxi No.5 People's Hospital. The inclusion criteria were as follows: (1) The patient showed mild brain symptoms at the first visit, with or without slight positive signs of stroke, and the NIHSS score $\leq 3$ points; ${ }^{3,19}$ (2) Patient received a brain MRI examination at the first visit. The MRI protocol included axial T1weighted image (T1WI), axial T2-weighted image (T2WI), axial fluid-attenuated inversion recovery (FLAIR), and diffusion weighted imaging (DWI). And MRI showed small infarcts; alternatively, DWI or FLAIR identified lacunar lesions or enhanced brain signals in the subcortical white matter, basal ganglia, or brainstem; (3) Imaging examination excluded intracranial hemorrhage or a nonvascular etiology of the patient's symptoms; (4) Stroke onset time was less than 72 hours; (5) The patient was above 18 years old; (6) The information on height, weight, waist circumference, blood pressure, blood lipids, fasting blood glucose (FBG), and medical history were complete; (7) The patient received aspirin therapy (100 mg daily); (8) The patient accepted 6 months of follow-up evaluation. The exclusion criteria were as follows: (1) Age < 18 years; (2) History of IS; (3) Cerebral hemorrhage or other active hemorrhagic diseases; (4) Brain tumor; (5) Traumatic brain injury, or other brain injuries; (6) Aneurysms or arteriovenous malformation (AVM) cases; (7) Dementia or mental illness; (8) Lack of MRI examination; (9) NIHSS score $>3$ at the first visit.

\section{Data Collection}

The following data were collected: the baseline data of the patient (age, height, weight, waist circumference, systolic 
blood pressure (SBP), diastolic blood pressure (DBP), FBG level, blood lipid level), medical history (hypertension, diabetes, dyslipidemia, metabolic syndrome), years of having hypertension, initial NIHSS score, symptoms (headache, dizziness, mild cognitive impairment (MCI)), MRI lesion location (anterior circulation artery (ACA), posterior circulation artery (PCA)), MRI results (number of lesions, the maximum diameters of lesions, size of infarcts).

Height, weight, and waist circumference were measured by standard methods. Body mass index (BMI) was calculated as weight $(\mathrm{kg}) / \mathrm{height}^{2}\left(\mathrm{~m}^{2}\right)$. FBG and blood lipids were measured in the laboratory. Hypertension referred to a clear medical record confirming the diagnosis of hypertension, or the two measurements of blood pressure under a calm state was greater or equal to 140/ $90 \mathrm{mmHg}$. Diabetes referred to a clear medical record confirming the diagnosis of diabetes, or FBG $\geq 7.0 \mathrm{mmol} /$ 1 , or random blood glucose $\geq 11.1 \mathrm{mmol} / 1$. Dyslipidemia referred to one or more of the following conditions: the total plasma cholesterol level was higher than $5.2 \mathrm{mmol} / \mathrm{l}$, the low-density lipoprotein cholesterol was higher than 3.4 $\mathrm{mmol} / \mathrm{l}$, the triglyceride level was higher than $1.7 \mathrm{mmol} / \mathrm{l}$, the high-density lipoprotein cholesterol was lower than $1.03 \mathrm{mmol} / 1$ in males or lower than $1.3 \mathrm{mmol} / \mathrm{l}$ in females. If the patient met three or more of the following items, he/ she would be diagnosed as metabolic syndrome: (1) waist circumference $\geq 90 \mathrm{~cm}$ for males and $\geq 80 \mathrm{~cm}$ for female; (2) triglycerides $>1.7 \mathrm{mmol} / 1$; (3) high-density lipoprotein cholesterol (HDL-C) $<1.03 \mathrm{mmol} / 1$ for male and $<1.3 \mathrm{mmol} / 1$ for female; (4) $\mathrm{SBP} \geq 130 \mathrm{mmHg}$, DBP $\geq 80 \mathrm{mmHg}$; (5) $\mathrm{FBG} \geq 5.6 \mathrm{mmol} / \mathrm{l}^{20}$ A neurologist evaluated the NIHSS score. All patients received brain MRIs within 24 hours of their first visit. MRI was performed using 1.5T equipment (Siemens). The MRI protocol included axial T1WI, axial T2WI, axial FLAIR, and DWI. A T1, T2, and FLAIR images were acquired using a multi-echo multi delay sequence. The synthetic MRI generates images using given repetition time (TR), echo time (TE), and inversion time (TI) based on the fitted data such as T1, T2, and proton density values of each voxel. Synthetic T1WIs were generated with a TR of $2500 \mathrm{~ms}$ and TE of $10 \mathrm{~ms}$. Synthetic T2WIs were generated with a TR of $6000 \mathrm{~ms}$ and TE of $110 \mathrm{~ms}$. Synthetic FLAIR was generated with a TR of $10,000 \mathrm{~ms}$, TE of $85 \mathrm{~ms}$, and TI of $2400 \mathrm{~ms}$. All MRIs were diagnosed by the same neuroradiologist and the same neurologist, who was not informed of the study. The examiner used FLAIR or DWI to assess whether there were high-intensity lesions specifically. The maximum diameter, number, location, and size of the lesions in each patient were recorded in detail.

The patients were followed up for 6 months to assess the occurrence of SVE. A neurologist collected the information over the phone. For patients who died during follow-up, the information was obtained from relatives or hospital records. SVE was defined as having one or more of the following conditions: transient ischemic attack (TIA), transient non-focal cerebrovascular symptoms, deterioration of cerebrovascular symptoms, recurrent infarction, and the mRS score $\geq 2$ during the follow-up. A total of 260 patients with MIS were included in this trial (see Figure 1).

\section{Statistical Analysis}

Continuous variables were expressed as mean \pm standard deviation (SD) when they followed a normal distribution and expressed as P50 (P25, P75) when they were nonnormally distributed. Categorical variables were expressed in frequency $(\%)$. Unpaired Student's $t$-test or MannWhitney nonparametric test was used for continuous variables in comparison between groups, and the Pearson chisquare test or Fisher exact test was used for categorical variables.

The splitSample function randomly divided the original dataset into a derivation set and validation set at a ratio of 3:1. Multivariable logistic regression analysis was conducted with 20 clinical candidate predictors as independent variables and SVE as the dependent variable. Backward stepwise selection based on minimal Akaike's information criterion (AIC) was used to eliminate redundant variables further. The resulting multivariate logistic regression model was used to calculate the odds ratio (OR) and 95\% confidence interval (CI) and build the final nomogram prognostic model.

We also plotted the nomogram of the prediction model, which could visually display each MIS patient's prediction results. Each variable is listed separately in the nomogram, and a corresponding number of points is assigned to a particular magnitude of the variable. Then, the cumulative score of all variables is matched to a scale of outcome. ${ }^{21}$ The calibration of the nomogram was assessed with a calibration curve. The receiver operating characteristic (ROC) curve of the prediction model was drawn, and the area under the curve (AUC) and its 95\% CI were calculated. Internal validation was performed using the dataset of the verification set. The AUCs between the derivation set and the validation 


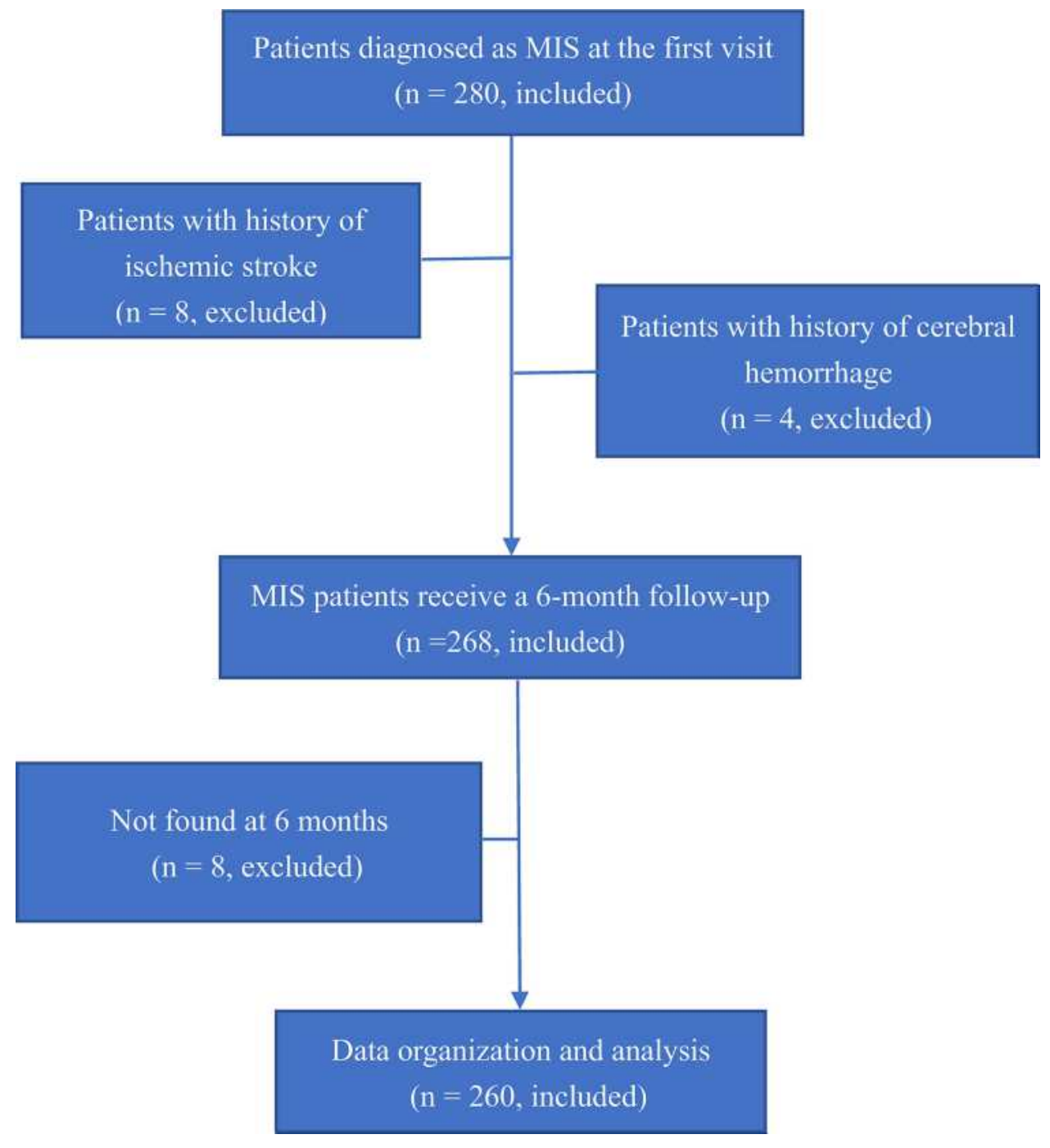

Figure I Flowchart of patient selection.

Abbreviation: MIS, minor ischemic stroke.

set were compared by the $\mathrm{z}$ statistics and Hanley and McNeil programs. $^{22}$ All statistical analysis was performed using R3.4.3 (http://www.R-project.org; software package: glmnet, pROC, rms), and all tests were two-sided. $\mathrm{P}<0.05$ was considered statistically significant.

\section{Results}

\section{Characteristics of the Modeling and Verification Sets}

Among the 260 enrolled MIS patients, 133 were male, and 127 were female, with an average age of $59.7 \pm 11.6$ years (37-85 years). SVE occurred in 51 cases (19.6\%) during the 6-month follow-up.

The 260 MIS patients were randomly divided into a derivation set (193 cases) and a verification set (67 cases).
The comparison of the baseline data between the two groups is shown in Table 1. There were no significant differences in age, gender, BMI, history of underlying diseases (hypertension, diabetes, dyslipidemia, metabolic syndrome), years of hypertension, SBP, DBP, and FBG between the two groups (all $\mathrm{P}>0.05$ ). NIHSS score, symptoms (headache, dizziness, $\mathrm{MCI}$ ), MRI lesion location (ACA, PCA), number of lesions, maximum diameter of lesions $(\mathrm{mm})$, infarct size $\left(\mathrm{mm}^{2}\right)$, and SVE incidence during follow-up were not significantly different between the two groups (all $\mathrm{P}>0.05$ ).

\section{Building a Predictive Model in the Derivation Set}

The results of the univariate analysis are shown in Table 2. The results suggested that age, diabetes, FBG, metabolic 
Table I The Baseline Characteristics of the Derivation Set and Verification Set

\begin{tabular}{|c|c|c|c|}
\hline & $\begin{array}{c}\text { Derivation } \\
\text { Set }(n=193)\end{array}$ & $\begin{array}{l}\text { Verification } \\
\text { Set }(n=67)\end{array}$ & $P$-value \\
\hline $\begin{array}{l}\text { Gender } \\
\text { Female } \\
\text { Male }\end{array}$ & $\begin{array}{l}92(47.7 \%) \\
101(52.3 \%)\end{array}$ & $\begin{array}{l}35(52.2 \%) \\
32(47.8 \%)\end{array}$ & 0.519 \\
\hline $\begin{array}{l}\text { Baseline data } \\
\text { Age (years) } \\
\text { BMI }\left(\mathrm{kg} / \mathrm{m}^{2}\right) \\
\text { SBP }(\mathrm{mmHg}) \\
\text { DBP }(\mathrm{mmH}) \\
\text { FBG }(\mathrm{mmol} / \mathrm{l})\end{array}$ & $\begin{array}{c}60.0 \pm 11.3 \\
23.9 \pm 2.8 \\
151.4 \pm 21.1 \\
95.8 \pm 11.6 \\
5.7 \pm 1.7\end{array}$ & $\begin{array}{c}58.8 \pm 12.4 \\
24.1 \pm 4.0 \\
151.8 \pm 21.9 \\
96.4 \pm 10.3 \\
5.9 \pm 2.0\end{array}$ & $\begin{array}{l}0.462 \\
0.716 \\
0.894 \\
0.724 \\
0.321\end{array}$ \\
\hline $\begin{array}{l}\text { Medical history } \\
\text { Hypertension } \\
\text { Diabetes Mellitus } \\
\text { Hyperlipidemia } \\
\text { Metabolic syndrome }\end{array}$ & $\begin{array}{l}169(87.6 \%) \\
14(7.3 \%) \\
111(57.5 \%) \\
76(39.4 \%)\end{array}$ & $\begin{array}{c}57(85.1 \%) \\
7(10.4 \%) \\
39(58.2 \%) \\
28(41.8 \%)\end{array}$ & $\begin{array}{l}0.602 \\
0.408 \\
0.921 \\
0.728\end{array}$ \\
\hline $\begin{array}{l}\text { Years of hypertension } \\
\text { (years) }\end{array}$ & I.0 (0.0-4.0) & $2.0(0.0-6.0)$ & 0.059 \\
\hline $\begin{array}{l}\text { NIHSS score } \\
0 \\
1 \\
2 \\
3\end{array}$ & $\begin{array}{c}155(80.3 \%) \\
33(17.1 \%) \\
5(2.6 \%) \\
0(0.0 \%)\end{array}$ & $\begin{array}{l}51(76.1 \%) \\
12(17.9 \%) \\
2(3.0 \%) \\
2(3.0 \%)\end{array}$ & 0.116 \\
\hline $\begin{array}{l}\text { Symptoms } \\
\text { Headache } \\
\text { Dizziness } \\
\mathrm{MCl}\end{array}$ & $\begin{array}{c}40(20.7 \%) \\
46(23.8 \%) \\
174(90.2 \%)\end{array}$ & $\begin{array}{l}\text { I5 (22.4\%) } \\
\text { I5 (22.4\%) } \\
61(91.0 \%)\end{array}$ & $\begin{array}{l}0.774 \\
0.810 \\
0.832\end{array}$ \\
\hline $\begin{array}{l}\text { Location of MIS } \\
\text { ACA } \\
\text { PCA }\end{array}$ & $\begin{array}{c}110(57.0 \%) \\
7(3.6 \%)\end{array}$ & $\begin{array}{c}34(50.7 \%) \\
4(6.0 \%)\end{array}$ & $\begin{array}{l}0.375 \\
0.412\end{array}$ \\
\hline $\begin{array}{l}\text { MRI findings } \\
\text { Number of lesions } \\
\text { Maximum diameters } \\
\text { of lesions ( } \mathrm{mm}) \\
\text { Size of infarcts }\left(\mathrm{mm}^{2}\right)\end{array}$ & $\begin{array}{l}8.0(4.0-12.0) \\
0.5(0.1-1.0) \\
5.0(3.0-6.0)\end{array}$ & $\begin{array}{c}5.0(4.0-11.5) \\
0.5(0.2-1.4) \\
5.0(3.5-7.0)\end{array}$ & $\begin{array}{l}0.522 \\
0.216 \\
0.075\end{array}$ \\
\hline SVE & 39 (20.2\%) & 12 (I7.9\%) & 0.683 \\
\hline
\end{tabular}

Notes: Mean \pm SD/Median (QI - Q3)/N (\%).

Abbreviations: BMI, body mass index; SBP, systolic blood pressure; DBP, diastolic blood pressure; FBG, fasting blood glucose; NIHSS, National Institutes of Health Stroke Scale; $\mathrm{MCl}$, mild cognitive impairment; MIS, minor ischemic stroke; ACA, anterior circulation artery; PCA, posterior cerebral artery; SVE, subsequent vascular events.

syndrome, MRI lesion number, and maximum diameter of lesions were statistically different between the patients with and without SVE (all $\mathrm{P}<0.05$ ), while gender, BMI, hypertension, years of hypertension, SBP, DBP, history of dyslipidemia, NIHSS score, symptoms, MRI lesion location, and infarct size were not statistically different (all $\mathrm{P}>0.05$ ).

We performed a binary multivariate logistic regression analysis using SVE as the dependent variable and the 20 indicators mentioned above as independent variables. Using the stepAIC method, we established the regression model and obtained independent factors, OR values, and 95\% CI for predicting SVE. Age, FBG, metabolic syndrome, number of lesions on MRI, and infarct size on MRI were independent factors for predicting SVE.

Prediction model formula: Logit $(\mathrm{P})=-14.57907+$ $0.13268 \times$ age $+0.77926 \times \mathrm{FBG}+1.06375 \times$ (metabolic syndrome $=1)+0.05442 \times$ number of lesions- $0.18792 \times$ infarct size. ( $\mathrm{P}$ is the probability of SVE)

The ROC curve of the prediction model in the derivation set was plotted. The AUC was 0.901 (95\% CI: 0.852-0.950), with a sensitivity of 0.795 , a specificity of 0.877 , a positive likelihood ratio of 6.443 , and a negative likelihood ratio of 0.234 . The ROC curve of the prediction model in the verification set was also plotted. The AUC was 0.897 (95\% CI: $0.810-0.984$ ), with a sensitivity of 0.833 , a specificity of 0.855 , a positive likelihood ratio of 5.729 , and a negative likelihood ratio of 0.195 . The AUC between the derivation set and validation set was similar (0.901 vs 0.897 ), with no significant difference $(\mathrm{P}=0.937)$ (see Figure 2).

\section{Nomogram and Calibration Curve of the Prediction Model}

We further plotted the nomogram (Figure 3A) and calibration curve (Figure 3B and C) of the SVE prediction model. The calibration curve showed that the prediction model had a good agreement between the predicted and observed derivation set and verification set values. We calculated the corresponding points of age, FBG, metabolic syndrome, number of lesions on MRI, and infarct size on MRI for MIS patients and then added all the points to obtain the total score. Then the nomogram was used to assess the risk of SVE based on the total score. For example, a 70-year-old (33 points) MIS patient, with an FBG of $8 \mathrm{mmol} / 1$ (33 points), no metabolic syndrome (0 points), 5 lesions on MRI (2 points), infarct size of $8 \mathrm{~mm}^{2}$ (16 points), the total score was 84 points, and the risk of SVE at 6 months was $40 \%$. The higher the risk value, the greater the possibility of SVE.

\section{Discussion}

In this study, a prognostic model was developed and validated. This nomogram was constructed based on age, 
Table 2 Univariate Analysis of SVE Incidence Based on Derivation Set

\begin{tabular}{|c|c|c|c|c|}
\hline SVE & No $(n=154)$ & Yes $(n=39)$ & OR $(95 \% \mathrm{Cl})$ & $P$-value \\
\hline \multicolumn{5}{|l|}{ Gender } \\
\hline Female & 71 (46.1\%) & 21 (53.8\%) & 1.0 & \\
\hline Male & $83(53.9 \%)$ & $18(46.2 \%)$ & $0.73(0.36,1.48)$ & 0.388 \\
\hline \multicolumn{5}{|l|}{ Baseline data } \\
\hline Age (years) & $57.9 \pm 11.0$ & $68.2 \pm 8.8$ & $1.10(1.05,1.14)$ & $<0.001$ \\
\hline BMI $\left(\mathrm{kg} / \mathrm{m}^{2}\right)$ & $23.9 \pm 2.8$ & $23.9 \pm 2.8$ & $0.99(0.87,1.13)$ & 0.893 \\
\hline $\mathrm{SBP}(\mathrm{mmHg})$ & $150.2 \pm 21.4$ & $156.1 \pm 19.4$ & $1.01(1.00,1.03)$ & 0.121 \\
\hline $\mathrm{DBP}(\mathrm{mmHg})$ & $96.4 \pm 12.0$ & $93.6 \pm 9.7$ & $0.98(0.95,1.01)$ & 0.185 \\
\hline FBG $(\mathrm{mmol} / \mathrm{l})$ & $5.3 \pm 0.8$ & $7.2 \pm 3.1$ & $2.48(1.67,3.67)$ & $<0.001$ \\
\hline \multicolumn{5}{|l|}{ Medical history } \\
\hline Hypertension & $169(87.6 \%)$ & 57 (85.1\%) & I.3। $(0.42,4.07)$ & 0.645 \\
\hline Diabetes Mellitus & 5 (3.2\%) & $9(23.1 \%)$ & $8.94(2.80,28.56)$ & $<0.001$ \\
\hline Hyperlipidemia & 84 (54.5\%) & 27 (69.2\%) & $1.88(0.89,3.97)$ & 0.101 \\
\hline Metabolic syndrome & 49 (31.8\%) & 27 (69.2\%) & $4.82(2.26,10.3 \mathrm{I})$ & $<0.001$ \\
\hline Years of hypertension (years) & $1.0(0.0-4.0)$ & $3.0(0.0-5.0)$ & $1.04(0.97,1.12)$ & 0.276 \\
\hline \multicolumn{5}{|l|}{ NIHSS score } \\
\hline 0 & $126(81.8 \%)$ & 29 (74.4\%) & 1.0 & \\
\hline I & 24 (I5.6\%) & $9(23.1 \%)$ & $1.63(0.69,3.87)$ & 0.269 \\
\hline 2 & $4(2.6 \%)$ & I (2.6\%) & $1.09(0.12,10.08)$ & 0.942 \\
\hline 3 & $0(0.0 \%)$ & $0(0.0 \%)$ & - & - \\
\hline \multicolumn{5}{|l|}{ Symptoms } \\
\hline Headache & $35(22.7 \%)$ & $5(12.8 \%)$ & $0.50(0.18,1.38)$ & 0.179 \\
\hline Dizziness & $33(21.4 \%)$ & $13(33.3 \%)$ & $1.83(0.85,3.96)$ & 0.122 \\
\hline $\mathrm{MCl}$ & $139(90.3 \%)$ & $35(89.7 \%)$ & $0.94(0.29,3.02)$ & 0.923 \\
\hline \multicolumn{5}{|l|}{ Location of MIS } \\
\hline ACA & 91 (59.1\%) & $19(48.7 \%)$ & $0.66(0.32,1.33)$ & 0.244 \\
\hline PCA & $5(3.2 \%)$ & $2(5.1 \%)$ & $1.61(0.30,8.63)$ & 0.578 \\
\hline \multicolumn{5}{|l|}{ MRI findings } \\
\hline Number of lesions & $5.5(3.2-10.0)$ & $12.0(8.0-17.5)$ & $1.05(1.02,1.09)$ & 0.002 \\
\hline Maximum diameters of lesions $(\mathrm{mm})$ & $0.5(0.1-1.0)$ & $1.0(0.2-2.4)$ & $1.43(1.15,1.77)$ & 0.001 \\
\hline Size of infarcts $\left(\mathrm{mm}^{2}\right)$ & $5.0(3.0-6.0)$ & $5.0(3.0-7.0)$ & $1.11(0.97,1.28)$ & 0.133 \\
\hline
\end{tabular}

Notes: Mean \pm SD/Median (QI - Q3)/N (\%).

Abbreviations: SVE, subsequent vascular events; BMI, body mass index; SBP, systolic blood pressure; DBP, diastolic blood pressure; FBG, fasting blood glucose; NIHSS, National Institutes of Health Stroke Scale; MCl, mild cognitive impairment; MIS, minor ischemic stroke; ACA, anterior circulation artery; PCA, posterior cerebral artery.

FBG, metabolic syndrome, number of MRI lesions, and infarct size, and it could predict the SVE incidence for MIS patients.

NIHSS score can predict the overall IS outcome. However, the difference in prognosis of patients with low NIHSS scores highlights the limitations of NIHSS as a prognostic factor in MIS patients. The NIHSS score may not capture some important defects that affect the functional outcome, such as reduced hand strength and agility, abnormal gait, and subtle non-dominant hemispheres, and abnormal cognitive executive function. In addition, the defects of MIS patients are usually subtle and may not be detected due to the lack of obvious damage. ${ }^{4,19}$

The current prediction models of IS mainly focus on evaluating the outcome of intravenous thrombolytic therapy, ${ }^{16,17,23}$ examining the safety of heparin and warfarin anticoagulation therapy, ${ }^{24,25}$ and predicting the risk of an asymptomatic cerebral hemorrhage after thrombectomy. ${ }^{26}$ Thrombolysis and thrombectomy are classic therapeutic interventions of IS. ${ }^{27}$ These IS patients who received intravenous thrombolysis, anticoagulation, and thrombectomy are relatively serious patients and are 


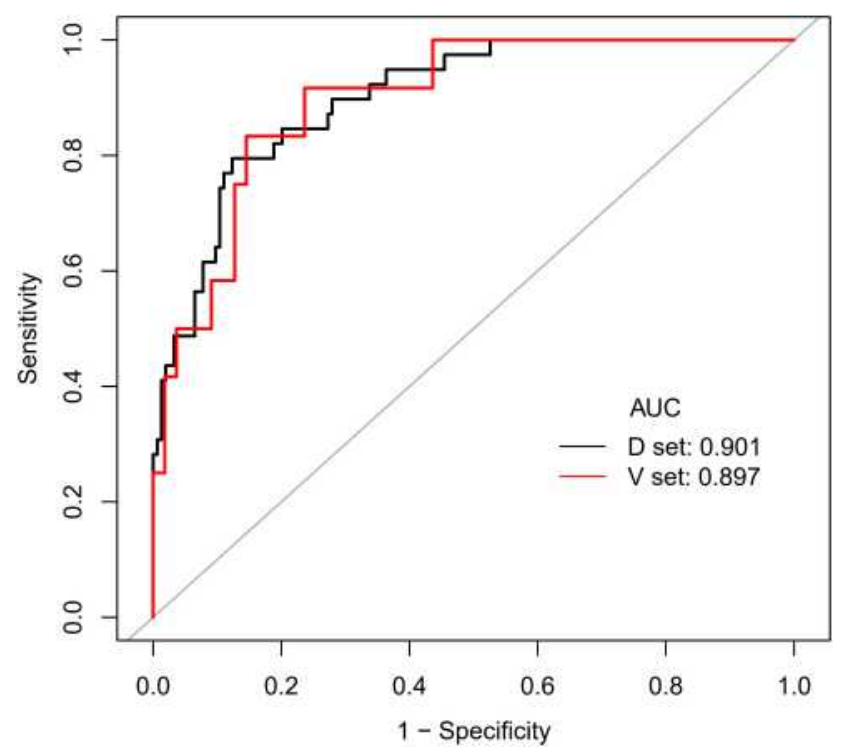

Figure 2 ROC curve of prediction model used for predicting 6-month SVE in Chinese MIS patients.

Abbreviations: ROC, receiver operating characteristic; SVE, subsequent vascular events; MIS, minor ischemic stroke.

not in the MIS category. NADE and COACHS nomograms are both developed and verified nomograms that can predict the poor prognosis of IS in the Chinese population. However, the NIHSS score of the included IS population ranged from 1 to 11 points, including MIS and non-MIS populations. ${ }^{13,14}$ MIS patients have mild initial symptoms and are more likely to be ignored. Therefore, we need more accurate evaluation models to predict the prognosis of MIS patients.

We are the first to show the visualized nomogram that predicts the individual probability of SVE at 6-month in Chinese MIS patients. Our results suggest that age, FBG, metabolic syndrome, number of lesions, and infarct size can predict the risk of SVE in MIS patients at 6-month. Unlike the prognostic score, our nomogram specifies the probability of a poor outcome (10\% to $90 \%)$. This personalized risk score calculation method can help us identify the patients with a high risk of adverse outcomes early.

This nomogram integrated the patient's age, FBG, metabolic syndrome, number of lesions, and infarct size and can be used to predict the poor prognosis of SVE at 6-month for each patient. We found that FBG was the strongest predictor among these five factors. When the patient's FBG reached $20 \mathrm{mmol} / 1$, the risk of SVE was higher than $80 \%$. Previous studies have shown that hyperglycemia is independently correlated with the 3-month mortality of acute ischemic stroke and mRS score $>2$ at 3-month (adjusted to age, NIHSS, and atrial fibrillation). ${ }^{28}$ FBG is a powerful predictor for the prognosis of patients with acute ischemic stroke. FBG, independent of admission glucose and $\mathrm{HbA} 1 \mathrm{c}$ intravenous thrombolytic therapy, is correlated with the 90-day adverse clinical outcome (mRS score, 3-6). ${ }^{29}$ Studies have shown that high blood glucose can promote inflammation, leading to decreased reperfusion, increased infarct size, neurological defects, cerebral edema, hemorrhagic transition, and patient death. ${ }^{30}$ The mechanism mentioned above can partially explain the unfavorable results that patients experience after IS.

We also found that the infarct size was negatively correlated with the SVE incidence. Studies have shown that patients with low mRS scores at 90 days may have smaller infarctions after endovascular therapy but have higher NIHSS scores at $24 / 48 \mathrm{~h}$. This may be related to whether the infarction is located in the eloquent brain or not. ${ }^{31}$ Ganesh et al found that patients with large posttreatment infarct volume (PIV) rarely developed serious adverse events. ${ }^{32}$ This result is consistent with our findings. The authors pointed out that, although the PIV was large, the sparing of lentiform nucleus was independently associated with good mRS scores.

Lentiform involvement may suggest a more proximal M1 segment middle cerebral artery or internal carotid artery involvement and potentially more white matter involvement than infarctions that spare the lentiform nucleus. $^{32,33}$ Therefore, a more detailed and precise analysis of lesion location can help us clarify the contribution of functional eloquence to stroke outcome.

Our study has several limitations. First of all, this is a retrospective case-control study. The data we collected was limited. Secondly, we excluded the patients with IS history, so the results were inappropriate for these patients. Thirdly, although it is enough to make statistically significant claims, having a more balanced dataset of SVE patients to non-SVE would be preferred. Finally, this model needs to be externally validated in different patient cohorts.

\section{Conclusion}

In conclusion, we constructed and verified a predictive model in predicting the 6-month SVE incidence of Chinese MIS patients. The nomogram contained five independent factors: age, FBG, metabolic syndrome, number of lesions, and infarct size on brain MRI. The model showed good diagnostic efficacy and high specificity, which could help clinicians stratify patients and provide optimal treatment. 
A

Points

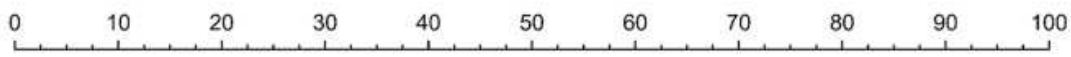

Age (years)

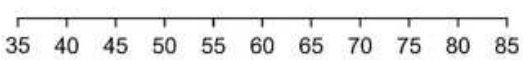

FBG (mmol/l)

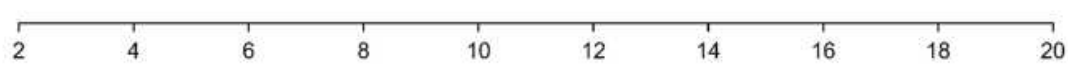

Metabolic

syndrome

$\overbrace{\text { no }}^{\text {yes }}$

Number of lesions

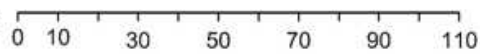

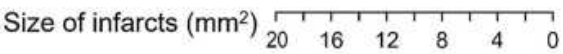

Total Points

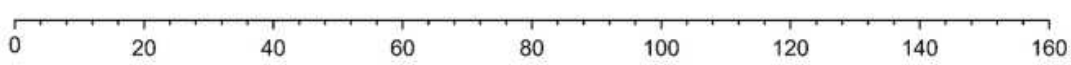

Linear Predictor

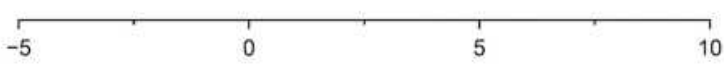

Risk of SVE
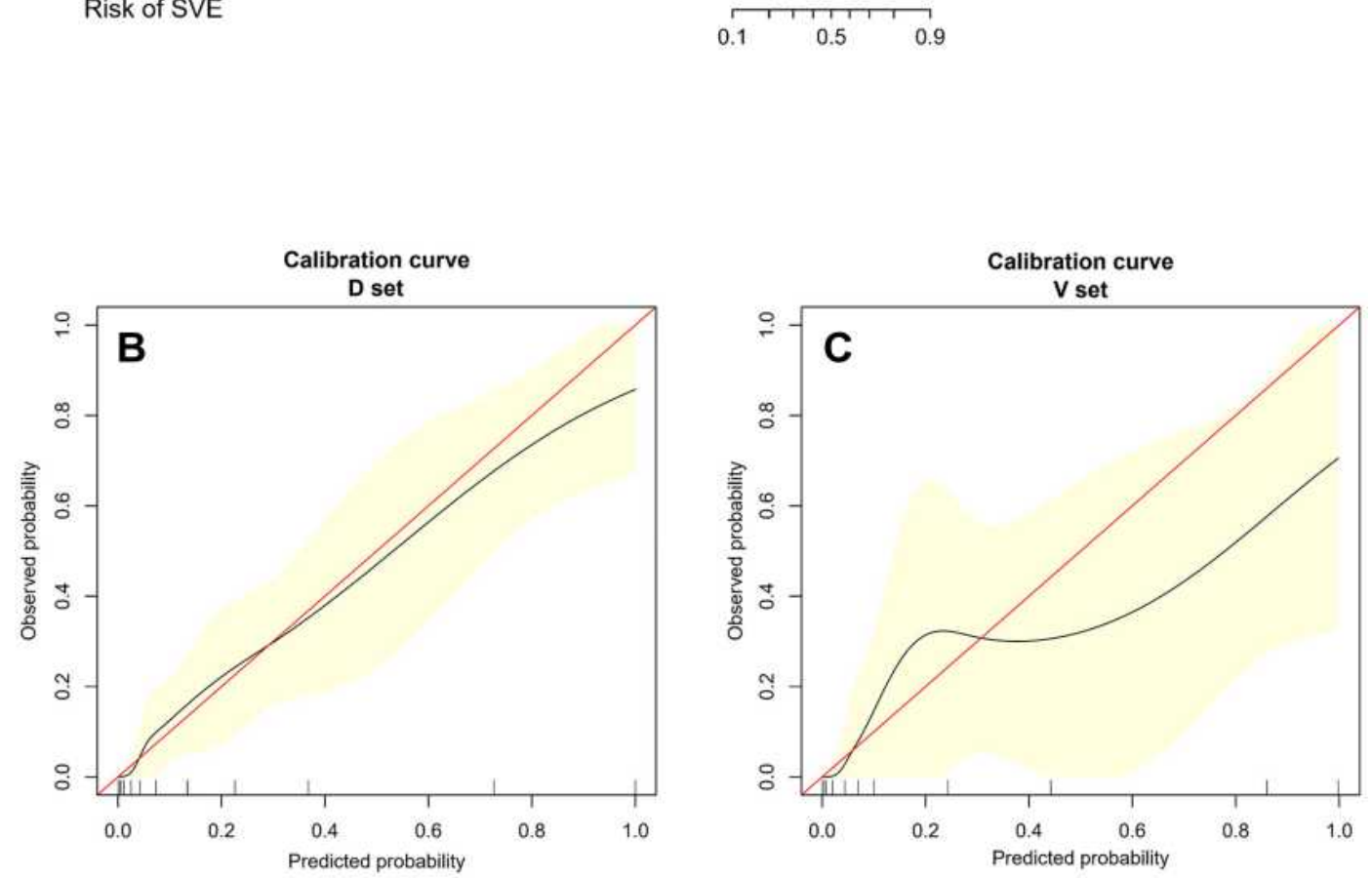

Figure 3 (A) The nomogram for predicting 6-month SVE in Chinese MIS patients. (B) The calibration curve of the model in the derivation set. (C) The calibration curve of the model in the verification set.

Notes: The horizontal axis is the predicted incidence of SVE, the vertical axis is the observed incidence of SVE; the red line on the diagonal is the reference line, indicating that the predicted value is equal to the actual value; the black line is the calibration curve, and the yellow areas on both sides represent $95 \% \mathrm{Cl}$. 


\section{Funding}

This research did not receive any specific grant from funding agencies in the public, commercial, or not-forprofit sectors.

\section{Disclosure}

The authors declare no conflicts of interest in this work.

\section{References}

1. Guan T, Ma J, Li M, et al. Rapid transitions in the epidemiology of stroke and its risk factors in China from 2002 to 2013. Neurology. 2017;89(1):53-61. doi:10.1212/WNL.0000000000004056

2. Wang W, Jiang B, Sun H, et al. Prevalence, incidence, and mortality of stroke in China results from a nationwide population-based survey of 480687 adults. Circulation. 2017;135(8):759. doi:10.1161/ CIRCULATIONAHA.116.025250

3. Fischer U, Baumgartner A, Arnold M, et al. What is a minor stroke? Stroke. 2010;41(4):661-666. doi:10.1161/STROKEAHA.109.572883

4. Rota E, Morelli N, Immovilli P, Cerasti D, Zini A, Guidetti D. "Minor" stroke: not a minor, still open question. J Thromb Thrombolysis. 2020;49(1):132-135. doi:10.1007/s11239-019-02001-w

5. Amarenco P, Lavallee PC, Tavares LM, et al. Five-year risk of stroke after TIA or minor ischemic stroke. $N$ Engl J Med. 2018;378 (23):2182-2190. doi:10.1056/NEJMoa1802712

6. van Wijk I, Kappelle LJ, van Gijn J, et al. Long-term survival and vascular event risk after transient ischaemic attack or minor ischaemic stroke: a cohort study. Lancet. 2005;365(9477):2098-2104. doi:10.1016/S0140-6736(05)66734-7

7. Amarenco P, Lavallee PC, Labreuche J, et al. One-year risk of stroke after transient ischemic attack or minor stroke. $N$ Engl $J$ Med. 2016;374(16):1533-1542. doi:10.1056/NEJMoa1412981

8. Tu WJ, Zhao SJ, Xu DJ, Chen H. Serum 25-hydroxyvitamin D predicts the short-term outcomes of Chinese patients with acute ischaemic stroke. Clin Sci. 2014;126(5):339-346. doi:10.1042/ CS20130284

9. Coutts SB, Modi J, Patel SK, et al. What causes disability after transient ischemic attack and minor stroke? Results From the CT And MRI in the Triage of TIA and minor Cerebrovascular Events to Identify High Risk Patients (CATCH) Study. Stroke. 2012;43 (11):3018-3022. doi:10.1161/STROKEAHA.112.665141

10. Balachandran VP, Gonen M, Smith JJ, DeMatteo RP. Nomograms in oncology: more than meets the eye. Lancet Oncol. 2015;16(4):e173180. doi:10.1016/S1470-2045(14)71116-7

11. Kasner SE. Clinical interpretation and use of stroke scales. Lancet Neurol. 2006;5(7):603-612. doi:10.1016/S1474-4422(06)70495-1

12. Banks JL, Marotta CA. Outcomes validity and reliability of the modified Rankin scale: implications for stroke clinical trials: a literature review and synthesis. Stroke. 2007;38(3):1091-1096. doi:10.1161/01.STR.0000258355.23810.c6

13. Sun C, Li X, Song B, et al. A NADE nomogram to predict the probability of 6-month unfavorable outcome in Chinese patients with ischemic stroke. BMC Neurol. 2019;19(1):274. doi:10.1186/ s12883-019-1464-6

14. Song B, Liu Y, Nyame L, et al. A COACHS nomogram to predict the probability of three-month unfavorable outcome after acute ischemic stroke in Chinese patients. Cerebrovasc Dis. 2019;47(1-2):80-87. doi: $10.1159 / 000497243$

15. Turcato G, Cervellin G, Cappellari M, et al. Early function decline after ischemic stroke can be predicted by a nomogram based on age, use of thrombolysis, RDW and NIHSS score at admission. $J$ Thromb Thrombolysis. 2017;43(3):394-400. doi:10.1007/s11239-016-1456-y
16. Cappellari M, Turcato G, Forlivesi S, et al. The START nomogram for individualized prediction of the probability of unfavorable outcome after intravenous thrombolysis for stroke. Int J Stroke. 2018;13 (7):700-706. doi:10.1177/1747493018765490

17. Song B, Chen X, Tang D, et al. External Validation of START nomogram to predict 3-month unfavorable outcome in Chinese acute stroke patients. $J$ Stroke Cerebrovasc Dis. 2019;28 (6):1618-1622. doi:10.1016/j.jstrokecerebrovasdis.2019.02.032

18. Sun W, Li G, Liu Z, et al. A nomogram for predicting the in-hospital mortality after large hemispheric infarction. BMC Neurol. 2019;19 (1):347. doi:10.1186/s12883-019-1571-4

19. Saber H, Saver JL. Distributional validity and prognostic power of the national institutes of health stroke scale in US administrative claims data. JAMA Neurol. 2020;77(5):606-612. doi:10.1001/jamaneurol.2019.5061

20. Grundy SM, Cleeman JI, Daniels SR, et al. Diagnosis and management of the metabolic syndrome: an American Heart Association/National Heart, Lung, and Blood Institute Scientific Statement. Circulation. 2005;112(17):2735-2752. doi:10.1161/ CIRCULATIONAHA.105.169404

21. Zlotnik A, Abraira V. A general-purpose nomogram generator for predictive logistic regression models. Stata J. 2015;15(2):537-546. doi:10.1177/1536867X1501500212

22. Hanley JA, McNeil BJ. The meaning and use of the area under a receiver operating characteristic (ROC) curve. Radiology. 1982;143(1):29-36. doi:10.1148/radiology.143.1.7063747

23. Yeo LLL, Chien SC, Lin JR, et al. Derivation and validation of a scoring system for intravenous tissue plasminogen activator use in Asian patients. J Stroke Cerebrovasc Dis. 2017;26(8):1695-1703. doi:10.1016/j.jstrokecerebrovasdis.2017.03.033

24. Yoo SH, Kwon SU, Jo MW, Kang DW, Kim JS. Age- and weight-adjusted warfarin initiation nomogram for ischaemic stroke patients. Eur J Neurol. 2012;19(12):1547-1553. doi:10.1111/j.14681331.2012.03772.x

25. Toth C, Voll C. Validation of a weight-based nomogram for the use of intravenous heparin in transient ischemic attack or stroke. Stroke. 2002;33(3):670-674. doi:10.1161/hs0302.104168

26. Cappellari M, Mangiafico S, Saia V, et al. IER-SICH nomogram to predict symptomatic intracerebral hemorrhage after thrombectomy for stroke. Stroke. 2019;50(4):909-916. doi:10.1161/STROKEAHA. 118.023316

27. Leng T, Xiong ZG. Treatment for ischemic stroke: from thrombolysis to thrombectomy and remaining challenges. Brain Circ. 2019;5 (1):8-11. doi:10.4103/bc.bc_36_18

28. Yoo DS, Chang J, Kim JT, et al. Various blood glucose parameters that indicate hyperglycemia after intravenous thrombolysis in acute ischemic stroke could predict worse outcome. PLoS One. 2014;9(4): e94364. doi:10.1371/journal.pone.0094364

29. Cao W, Ling Y, Wu F, Yang L, Cheng X, Dong Q. Higher fasting glucose next day after intravenous thrombolysis is independently associated with poor outcome in acute ischemic stroke. $J$ Stroke Cerebrovasc Dis. 2015;24(1):100-103. doi:10.1016/j. jstrokecerebrovasdis.2014.07.029

30. Clark ME, Payton JE, Pittiglio LI. Acute ischemic stroke and hyperglycemia. Crit Care Nurs Q. 2014;37(2):182-187. doi:10.1097/CNQ.0000000000000015

31. Al-Ajlan FS, Al Sultan AS, Minhas P, et al. Posttreatment infarct volumes when compared with 24-hour and 90-day clinical outcomes: insights from the REVASCAT Randomized Controlled Trial. AJNR Am J Neuroradiol. 2018;39(1):107-110. doi:10.3174/ajnr.A5463

32. Ganesh A, Menon BK, Assis ZA, et al. Discrepancy between post-treatment infarct volume and 90-day outcome in the ESCAPE randomized controlled trial. Int J Stroke. 2020;17474993020929943.

33. Russmann H, Vingerhoets F, Ghika J, Maeder P, Bogousslavsky J. Acute infarction limited to the lenticular nucleus: clinical, etiologic, and topographic features. Arch Neurol. 2003;60(3):351-355. doi:10. 1001/archneur.60.3.351 


\section{Publish your work in this journal}

Therapeutics and Clinical Risk Management is an international, peerreviewed journal of clinical therapeutics and risk management, focusing on concise rapid reporting of clinical studies in all therapeutic areas, outcomes, safety, and programs for the effective, safe, and sustained use of medicines. This journal is indexed on PubMed Central, CAS,
EMBase, Scopus and the Elsevier Bibliographic databases. The manuscript management system is completely online and includes a very quick and fair peer-review system, which is all easy to use. Visit http://www.dovepress.com/testimonials.php to read real quotes from published authors. 